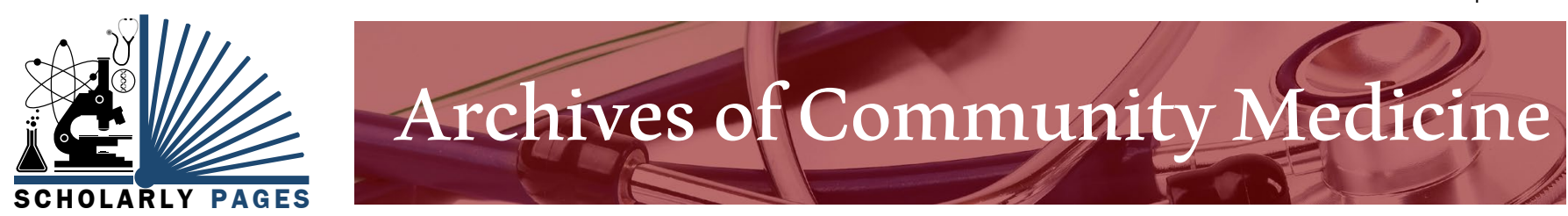

\title{
A Comparison of Prescribing Practices for the Treatment of Malaria in Public and Private Health Facilities in Southeast Nigeria
}

\author{
Chinyere Okeke ${ }^{1,2^{*}}$, BSC Uzochukwu ${ }^{1,2}$, and Obionu Ifeoma ${ }^{1}$ \\ ${ }^{1}$ Department of Community Medicine, Faculty of Medical Sciences, College of Medicine, University of Nigeria, \\ Enugu, Nigeria \\ ${ }^{2}$ Health Policy Research Group, Department of Pharmacology and Therapeutics, College of Medicine, University \\ of Nigeria, Enugu, Nigeria
}

\begin{abstract}
Introduction: Polypharmacy and irrational prescription of antimalaria drugs and injections are indications of irrational prescribing practices and may lead to drug resistance. The study determined the prescribing practices for the treatment of malaria in public and private health centres in Enugu urban of south east Nigeria. Methodology: This was a descriptive, cross-sectional study carried out in Enugu urban, southeast Nigeria in December 2014. One hundred prescription records were collected from four health centers and forty from ten health centers to determine the percentage of antimalaria drugs appropriately prescribed; percentages of prescribed injection and antibiotics and average number of drugs per case.

Results: Antimalaria drugs were prescribed in wrong doses in private and public health facilities (25 vs. $39.3 \%$ ). ACTs were prescribed for malaria in public than private (65 vs. $44 \%)$. However, all the ACTs prescribed in both public and private facilities were in their trade names rather than generic names. AMT was still prescribed in both facilities but more in private than public facilities (36 vs. 18\%). More injections (87.7 vs. $45.0 \%)$ were prescribed in private than public health facilities $(\mathrm{p}<0.05)$. More antibiotics $(64.3$ vs. $23.0 \%)$ were prescribed in private than public health facilities $(\mathrm{p}<0.05)$. The public health centers had an average of 6.2 drugs per prescription against 3.2 in the private facilities $(\mathrm{p}<0.05)$.

Conclusion: Prescribing practices by both private and public health workers are irrational and these are more with the private. Strategies to ensure appropriate and rational drug prescribing among health workers such as prescription education are recommended.
\end{abstract}

\section{Introduction}

Medicines play an integral part of healthcare delivery. However, they are expensive commodities and account for a significant proportion of overall health expenditure in most countries. The World health organization (WHO) estimates that more than half of all medicines are inappropriately prescribed, dispensed or sold [1]. Irrational use of medicines is a major challenge facing many health systems across the world [2]. The problem of irrational medicine use is known to be worse in developing countries with weak health systems, where mechanisms for routine monitoring of medicine use are often not well developed or are at times non-existent [3]. Such practices are likely to lead to poor health delivery that may put patients at risk and result in wastage of scarce resources that could have been used to tackle other pressing health needs [3].

Irrational prescribing has been found to be caused by health systems factors like work place heavy patient load; lack of enforced regulations; pressure of promotional

*Corresponding author: Chinyere Okeke, Department of Community Medicine, Faculty of Medical Sciences, College of Medicine, University of Nigeria, Enugu, Nigeria, E-mail: chinyereokeke83@yahoo.com

Received: June 29, 2017; Accepted: June 06, 2018; Published online: June 08, 2018

Citation: Okeke C, Uzochukwu BSC, Ifeoma O (2018) A Comparison of Prescribing Practices for the Treatment of Malaria in Public and Private Health Facilities in Southeast Nigeria. Arch Community Med 1(1):1-5

Copyright: (c) 2018 Okeke C, et al. This is an open-access article distributed under the terms of the Creative Commons Attribution License, which permits unrestricted use, distribution, and reproduction in any medium, provided the original author and source are credited. 
Citation: Okeke C, Uzochukwu BSC, Ifeoma O (2018) A Comparison of Prescribing Practices for the Treatment of Malaria in Public and Private Health Facilities in Southeast Nigeria. Arch Community Med 1(1):1-5

activities; discrepancy of lab results; lack of supervision, monitorind and evaluation [4]. Other factors identified are prescribers and dispensers issues like inadequate knowledge and skills, lack of evidence based practices; presure to prescribe and dispense; lack of medication information; lack of continous professional development [5]. While patient factors like patient/relatives demands/ exepectations; wrong health beliefs; cultural practices; lack of health education.

Drug use problems such as polypharmacy and irrational prescription of antimalaria and injections are indications of irrational prescribing practices and may lead to drug resistance [6-10]. It has also been seen to lead to medicine stock outs, eroding patients confidence in the health systems and leading to cause of loss of public and personal resources [11].

Resistance has arisen to all classes of anti-malaria drugs. This has increased the global malaria burden and is a major threat to malaria control. Studies have found that widespread and indiscriminate use of anti-malaria drugs places a strong selective pressure on malaria parasites to develop high levels of resistance $[10,12]$.

Anti-malaria drug resistance is therefore defined as the ability of a parasite strain to survive and/or multiply despite the administration and absorption of a drug given in doses equal to or higher than those usually recommended but within tolerance of the subject, the drug in question having gained access to the parasite or the infected red blood cell for the duration of the time necessary for its normal action [13-17].

Studies have shown that one of the influences on development of anti-malarial drug resistance includes overall drug pressure, among others [18]. This has also been considered the single most important factor in the development of resistance [6,19]. Reliance on presumptive treatment can also facilitate the development of anti-malaria drug resistance.

Interventions aimed at preventing drug resistance, per se, generally focus on reducing overall drug pressure through more selective use of drugs; improving the way drugs are used through improving prescribing, follow-up practices, and patient compliance; or using drugs or drug combinations which are inherently less likely to foster resistance or have properties that do not facilitate development or spread of resistant parasites [13,20]. In other words, interventions aimed at promoting the rational use of drugs.

Rational use of drugs requires that patients receive medications appropriate to their clinical needs, in doses that meet their own individual requirements for an adequate period of time, and the lowest cost to them and their community [21]. The definition implies that ratio- nal use of drugs, especially rational prescribing should meet certain criteria. Unfortunately, in the real world, the prescribing pattern does not always conform to criteria and can be classified as inappropriate or irrational prescribing.

Irrational prescribing may be regarded as "pathological" prescribing [21], as occurs with overuse of antibiotics and anti-diarrheals, indiscriminate use of injections, multiple drug prescriptions (polypharmacy), minerals and tonics for malnutrition.

The impact of this irrational use of drugs can be seen in many ways: Reduction in the quality of drug therapy leading to increased morbidity and mortality, Waste of resources leading to reduced availability of other vital drugs and increased costs. Increased risk of unwanted affects such as adverse drug reactions and the emergence of drug resistance, Psychosocial impacts, such as when patients come to believe that there is "a pill for every ill". This may cause an apparent increased demand for drugs [10].

The availability of drugs is one of the most visible symbols of quality care to consumers as in a study it was shown that the patients' visits dropped when health facilities ran out of commonly used drugs [22].

Consumers perception of expertise of health workers is linked to their prescribing rate and the greater the number of drugs prescribed, the higher the perceived expertise of the health worker [23]. Some authors have recommended just two drugs per prescription [24] and that justification for more drugs than this should be required because of the increased risk of drug interactions [25]. Poor prescribing practices often result in inappropriate use of drugs.

In some countries, high numbers of drugs per prescription have been found [26]. Studies revealed that the average number of drugs prescribed in public sector facilities in most developing countries ranged from 1.3 to 2 drugs between 1990 and 1992. In Indonesia, Nigeria and Ghana the figures differed significantly from this average. Results show that in Indonesia, the average number of drugs per case was 3.3, in Nigeria 3.8 and in Ghana 4.3 [21]. When the number of drugs per case was broken down by diagnosis, very little change was noted showing that the general pattern of polypharmacy occurs almost independent of diagnosis. Evidence exists in Nigeria that health personnel tend to embark on polypharmacy in their attempts to treat a number of possible diseases simultaneously [27].

Reports have indicated that unnecessary use of medicines can stimulate inappropriate patient demand and over prescribing quickly exhausts stocks of medicines in public health facilities, leading to patients' loss of confi- 
Citation: Okeke C, Uzochukwu BSC, Ifeoma O (2018) A Comparison of Prescribing Practices for the Treatment of Malaria in Public and Private Health Facilities in Southeast Nigeria. Arch Community Med 1(1):1-5

Table 1: Prescription analysis.

\begin{tabular}{|c|c|c|c|}
\hline Indicators & Private & Public & Chi square (p-value) \\
\hline Percentage of antimalaria drugs were prescribed in wrong doses & $25 \%$ & $39.3 \%$ & $3.10[0.0780900]$ \\
\hline Average percentage of prescriptions with an injection & 87.7 & $45.0 \%$ & $21.07[0.0000044]$ \\
\hline Average percentage of prescriptions with one or two antibiotics & 64.3 & $23.0 \%$ & $22.15[0.0000025]$ \\
\hline Average number of drugs per prescription (SD) & 3.2 & 6.2 & $0.74[0.03906743]$ \\
\hline
\end{tabular}

dence in the health-care system and reduced attendance [28]. A situation that a private hospital can ill afford with the usual profit orientation of such facilities as against the public health facilities with a different orientation.

The rational use of drugs is potentially more problematic at lower levels of the health services like the Primary Health Centers where supervision and controls even in the best of conditions is less rigorous $[29,30]$. The private health facilities even more, with little or no supervision or control.

The prescribing practices in the public and private health facilities in the treatment of malaria is not known in Nigeria. The aim of this study is to compare the prescribing practices for the treatment of malaria in public and private health facilities in southeast Nigeria.

\section{Methods}

\section{Study area}

This study took place in Enugu urban, southeast Nigeria. Enugu state has a total population of about 3,257,298 people [31]. Enugu urban is the capital city of Enugu state. It comprised of Enugu north and parts of Enugu south and east LGAs. It houses about five public tertiary health institutions, good numbers of private hospitals and primary health centers, and large number of drug retailers scarttered all over the place. There are also good roads and these are indications of physical access to the health facilities in all seasons of the year.

\section{Study design}

This was a descriptive, cross-sectional study that took place in Enugu urban, southeast Nigeria.

\section{Data collection}

Treatment records from Primary health centers were randomly selected retrospectively; prescriptions from the private clinics were collected prospectively using surrogate patients. One hundred prescription records were collected from four health centers ( 25 per health center), and four prescriptions each were collected from 10 private clinics (40 total).

\section{Data analysis}

Data entry and analysis was done using SPSS version 20. For each of the prescriptions, the following variables were analysed: The percentage of prescriptions with an-
Table 2: Table of characteristics of subjects whose prescriptions were used for the study.

\begin{tabular}{|l|l|l|}
\hline Variable & $\begin{array}{l}\text { Public health } \\
\text { facility (N= 100) }\end{array}$ & $\begin{array}{l}\text { Private health } \\
\text { facility (N = 40) }\end{array}$ \\
\hline Age (Years) & Frequency (\%) & Frequency (\%) \\
\hline $0-10$ & $27(27)$ & $10(25.0)$ \\
$11-20$ & $18(18)$ & $6(15.0)$ \\
$21-30$ & $32(32)$ & $11(27.5)$ \\
$31-40$ & $9(9)$ & $5(12.5)$ \\
$41-50$ & $6(6)$ & $3(7.5)$ \\
$51-60$ & $3(3)$ & $5(12.5)$ \\
$61-70$ & $5(5)$ & $0(0)$ \\
\hline Mean $\pm(S D)$ years & $24.3(24.3)$ & $21.7(24.2)$ \\
\hline Sex & & \\
\hline Male & $21(21.0)$ & $9(22.5)$ \\
\hline Female & $79(79.0)$ & $31(77.5)$ \\
\hline
\end{tabular}

timalaria drugs appropriately prescribed; percentages of prescribed injection and antibiotics and the average number of drugs per case.

\section{Results}

The mean age of the respondents was found to be 24.3 years and there were more females than males in both gropus: $79 \%$ and $77.5 \%$ respectively as shown in Table 2.

Antimalaria drugs were prescribed in wrong doses in both private and public health facilities ( 25 vs. $39.3 \%)$ respecticely as shown in Table 1.

More injections ( 87.7 vs. $45.6 \%$ ) and more antibiotics (64.3 vs. $23.4 \%$ ) were prescribed in private than public health facilities $(\mathrm{p}<0.05)$ and both were found to be statistically significant as shown in Table 1 . Polypharmacy and irrational prescription of vitamins and other combination preparations was common in both public and private facilities. However, the public health centers had an average of 6.2 drugs per prescription against 3.2 in the private facilities. In general, prescriptions were significantly poorer in the private health facilities compared to the public.

\section{Discussion}

The study has shown that prescribing practices in the study area are irrational, the situation in the private health facilities is poorer than that in the public health facilities. This may be attributable to the different orientations of these sectors, the private facilities being more 
Citation: Okeke C, Uzochukwu BSC, Ifeoma O (2018) A Comparison of Prescribing Practices for the Treatment of Malaria in Public and Private Health Facilities in Southeast Nigeria. Arch Community Med 1(1):1-5

profit oriented. Injection use is a simple indicator of therapeutic practices [21], and this may lead to irrational prescribing and drug resistance. This study found that more chloroquine injections ( 87.7 vs. $45.6 \%$ ) were used in the private than public health facilities. The pressure towards consumer satisfaction in private health facilities, may be the reason for this high use as prescribers tend to believe that patients expect and are satisfied by receiving injections and that some patients would ask for injections even when this has not been prescribed. However, this high use could also be attributable to the fact that in health care market, patients may not be wholly informed and so may depend on the decisions of their providers, and this is more with the private who are more profit oriented. It can result also from socio-cultural factors such as patients' demand for drugs especially in the private health facilities. In a study in Indonesia, almost half of the under-fives and more than $70 \%$ of the over-five age group in the study, received at least one injection and when injection use was broken down by diagnosis, overuse was found to be common for many illnesses [21].

The source of irrational use may be from the prescriber, [32-34] as prescribers tend to believe that patients expect and are satisfied by receiving injections [21] or the consumer $[30,31]$ for some patients do ask for injections. However, many dislike injections but accept them because of the belief that, "the doctor knows best".

Antibiotics use being another indicator of drug use and commonly used in developing countries due to the high load of infection, in this study, was found to be high $64.3 \%$ in the private facilities and $23.4 \%$ found in the public facilities. This was higher than the range of 25$40 \%$ given for most countries [19]. Excessive antibiotic use leads to resistance. Antibiotics are commonly used in developing countries due to the high load of infection, often as broad spectrum, often without the guidance of laboratory investigations.

Polypharmacy, is a problem in the study area. The number of drugs per case or per episode describes the pattern of polypharmacy, where more than one drug is prescribed (often unnecessarily) for a condition. In one study in Indonesia, the occurrence of polypharmacy or multiple prescribing was obvious, where the average number of drugs per case was 3.8 both for under-fives and for the over-five age group [17]. The average number of drugs prescribed in public facilities in developing countries has been noted as 1.3-2 drugs per prescripton, for Ghana and Nigeria, the values were much higher, 3.8 drugs per prescription for Nigeria [17]. This study however showed the public health facilities as having an average of 6.2 drugs per prescription against 3.2 in the private facilities, indicating that the practice of polypharmacy is greater in the public facilities and may be on the increase. Polypharmacy and irrational prescription of vitamins and other combination preparations are common in both public and private facilities, the private facilities prescribing more injections and antibiotics than the public health facilities. This practice of polypharmacy and irrational prescribing could be linked to the health workers' knowledge and training. Some other studies in Nigeria found that the greater the number of drugs prescribed, the higher the perceived expertise of the health worker [19]. The higher occurrence in public than private found in this study may be linked to the findings of [35], where PHC workers tend to over prescribe to quickly exhaust stocks of medicines.

Lack of continuing education and training like; inappropriate role models, the lack of objective drug information, the generalization of limited experience, and also misleading beliefs about drugs efficacy are likely to lead irrational prescribing.

The requirements to be fulfilled if the process of prescribing is appropriately followed will include: Steps in defining the patient's problems (or diagnosis); in defining effective and safe treatments (drugs and non-drugs); in selecting appropriate drugs, dosage and duration; in writing a prescription; in giving patients adequate information; and in planning to evaluate treatment responses.

Establishing a mandated multidisciplinary national body to coordinate policies on medicine use as part of a national medicines policy; Formulating and using evidence-based clinical guidelines for training and supervision; Selecting essential medicines lists based on treatments of choice; Setting up drug and therapeutics committees in larger hospitals; Promoting problem-based training in pharmacotherapy as part of undergraduate training; Making continuing in-service medical education a requirement of licensure; Promoting systems of supervision, audit and feedback in institutional settings; Providing independent information about medicines; Promoting public education about medicines; Eliminating perverse financial incentives that lead to irrational prescribing; Drawing up and enforcing regulation, including that of promotional activities; serving sufficient governmental expenditure to ensure equitable availability of medicines and health personnel.

Multifaceted, coordinated interventions are more effective than single interventions in changing prescribing practices by both public and private sector health providers. This should include four intervention strategies to improve drug use [32], and these are educational, managerial, financial, and regulatory interventions. They should be based on detailed analyses of existing problems and take into account financial incentives. Intervention strategies should reflect local needs and may include me- 
Citation: Okeke C, Uzochukwu BSC, Ifeoma O (2018) A Comparison of Prescribing Practices for the Treatment of Malaria in Public and Private Health Facilities in Southeast Nigeria. Arch Community Med 1(1):1-5

dia campaigns, treatment guidelines, package labeling, essential drug lists, flow charts and diagnostic cards and individual and group feedback on practice. Studies in India [34] and China [36] have also revealed that these sorts of interventions to improve prescribing practices and drug use were effective.

\section{Conclusions}

Prescribing practices by both private and public health workers are irrational and these are more with the private. Strategies to ensure appropriate and rational drug prescribing among health workers in both private and public health facilities such as prescription education are recommended. More detailed studies (for example by Focus Group discussions and structured interviews) should be undertaken to find out reasons for the over prescription and to develop future interventions to correct this.

\section{References}

1. (2011) World Medicines Situation Report. World Health Organization, Switzerland.

2. (2004) The World Medicines Situation. World Health Organization, Switzerland.

3. Ofori Asenso R, Brhlikova P, Pollock AM (2016) Prescribing indicators at primary health care centers within the WHO African region: A systematic analysis (1995-2015). BMC Public Health 16: 724.

4. WHO (2010) Rational use of medicines. Fact sheet $\mathrm{N}^{\circ} 338$.

5. (2007) Assessment of national pharmaceutical sector. $\mathrm{MOH}, \mathrm{DGoP}$, North Sudan.

6. Peter B Bloland (2001) Drug resistance in Malaria. Malaria epidemiology branch, center for disease control and prevention Chamblee, GA, USA.

7. Fulton MM, Allen ER (2005) Polypharmacy in the elderly: A literature review. J Am Acad Nurse Pract 17: 123-132.

8. (1993) How to investigate drug use in health facilities: Selected drug use indicators - EDM Research Series No. 007. WHO: 92.

9. Haider SI, Johnell K, Weitoft GR, et al. (2009) The influence of educational level on polypharmacy and inappropriate drug use: A register-based study of more than 600,000 older people. J Am Geriatr Soc 57: 62-69.

10. Haider SI, Johnell K, Thorslund M, et al. (2007) Trends in polypharmacy and potential drug-drug interactions across educational groups in elderly patients in Sweden for the period 1992-2002. Int J Clin Pharmacol Ther 45: 643-653.

11. Singh $P$, Tiwari $P$ (2001) Drug utilisation studies: The need for a common protocol. Indian Express Newspaper.

12. (2012) Managing access to medicines and health technology. MSH.

13. WHO (2006) Guidelines for the treatment of Malaria.

14. Bruce Chwatt LJ, Black RH, Canfield CJ, et al. (1986) Chemotherapy of malaria. World Health Organization, Geneva.

15. WHO (2010) Drug resistance: Malaria. 9.

16. Nicolas WJ (2004) Antimalarial drug resistance. J Clin Invest 113: 1084-1092.

17. Wernsdorfer WH (1994) Epidemiology of drug resistance in malaria. Acta Trop 56: 143-156.

18. Seppana H, Klaukka T, Viapio Varkila J, et al. (1997) The effect of changes in the consumption of macrolide antibiotics on erythromycin resistance in Group A streptococci in Finland. Finnish Study Group for Antimicrobial Resistance. N Engl J Med 337: 441-446.

19. Bauchner H, Pelton SI, Klein JO (1999) Parents, physicians, and antibiotic use. Pediatrics 103: 395-401.

20. Schwartze C, Harrison E, Garland O (2007) Preventing substance disorders in children and youths. Journal of Child Health Policy 2.

21. http://archives.who.int/PRDUC2004/RDUCD/Session_ Guides/problems_of_irrational_drug_use.htm

22. (1994) The importance of pharmaceuticals and essential drug programs. In: Better Health in Africa: Experience and Lessons Learned. World Bank, Washington DC.

23. Uzochukwu BSC, Onwujekwe OE, Akpala CO (2002) Effect of the Bamako Initiative Drug Revolving Fund on availability and rational use of essential drugs in primary health care facilities in south east Nigeria. Health Policy Plan 17: 378383.

24. Nes SA (1990) Principles of therapeutics. In: Gilman GA, Rali NT, The pharmacological basis of therapeutics. Pergoman Press, New York, 62-83.

25. Falkenberg T, Nguyen TB, Larsson M, et al. (2000) Pharmaceutical sector in transition-a cross sectional study in Vietnam. Southeast Asian J Trop Med Public Health 31: 590-597.

26. (1992) World Bank. Pharmaceutical expenditures and cost recovery schemes in Sub-Saharan Africa. Technical working paper 4. African Technical Department, Population, Health, and Nutritional Division. World Bank, Washington DC.

27. Executive board 115 (2005) Rational use of medicines by prescribers and patients: Report by the Secretariat. World Health Organization.

28. Ross J (2007) Illicit drug use in Australia: Epidemiology, use pattern and associated harm. ( $2^{\text {nd }}$ edn), National Drug and Alcohol research Centre, Australia.

29. Health and livelihood needs of residents of informal settlements in Nairobi city. African Population and Health Research Center. Occasional Study Report 2002.

30. (2004) Nigerian demographic and health surveys 2003. National Population Commission. Federal Republic of Nigeria and ORC Macro, Calverton, Maryland USA.

31. Laing RO (1990) Rational drug use: An unsolved problem. Trop Doct 20: 101-103.

32. Gilson L, Jaffar S, Mwankusye S, et al. (1993) Assessing prescribing practices: A Tanzanian example. International Journal of Health Planning and Management 8: 37-58.

33. Hogerzeil HV, Ross Degnan R, Laing R, et al. (1993) Field tests for rational drug use in twelve developing countries. The Lancet 342: 1408-1410.

34. Greenhalgh T (1987) Drug prescriptions and self-medication in India: An exploratory survey. Soc Sci Med 25: 307318.

35. Kshirsagar MJ, Langade D, Patil S, et al. (1998) Prescribing patterns among medical practitioners in Pune India. Bull World Health Organ 76: 271-275.

36. Hardon AP (1987) The use of modern pharmaceuticals on a Filipino village doctors' prescriptions and self medication. Soc Sci Med 25: 277-292. 